\title{
Optical and transport properties of InSb thin films grown on GaAs by metalorganic chemical vapor deposition
}

\author{
Tzuen-Rong Yang ${ }^{\mathrm{a}}$, Yukun Cheng ${ }^{\mathrm{a}}$, Jyun-Bi Wang ${ }^{\mathrm{b}}$, Zhe Chuan Feng ${ }^{\mathrm{b}, *}$ \\ a Department of Physics, National Taiwan Normal University, Taipei, 116 Taiwan, ROC \\ ${ }^{\mathrm{b}}$ Graduate Institute of Electro-Optical Engineering and Department of Electrical Engineering, College of Electrical Engineering, \\ National Taiwan University, Taipei, 106-17 Taiwan, ROC
}

Available online 19 August 2005

\begin{abstract}
Optical and transport properties of InSb thin films grown on GaAs by metalorganic chemical vapor deposition (MOCVD) have been investigated by far-infrared (FIR) reflectance spectroscopy. The lattice vibration behaviors of a series of $\mathrm{MOCVD} \mathrm{InSb} / \mathrm{GaAs}(100)$ materials grown under different growth conditions were studied. Effects of III-V source ratios on the films crystalline quality were examined. Two additional weak modes in the wavenumber regions of $210-240 \mathrm{~cm}^{-1}$ were observed and they appeared more prominent at low temperatures. Interference fringe effects modify the FIR reflectance band of the GaAs substrate, which are related to the uniformity of film thickness and crystalline perfection. The dielectric constant, phonon modes and other optical parameters, as well as transport properties including carrier concentration, mobility, effective mass were calculated theoretically and compared with experimental results. The obtained distribution values of the InSb LO phonon mode frequency, line width, relative integrated intensity ratio between the forbidden and defect-related TO phonon and the allowed LO mode are adopted as figures of merit for the quality of the InSb films. The electrical transport properties of carrier concentration, mobility, and effective mass as well as the dielectric constant of these films have been determined by optical method non-destructively.
\end{abstract}

(C) 2005 Elsevier B.V. All rights reserved.

Keywords: Metalorganic chemical vapor deposition (MOCVD); Characterization; InSb; GaAs; Fourier transfer infrared spectroscopy

\section{Introduction}

InSb has important applications in infrared, optical, microwave, and millimeter-wave devices [1-4]. It is a narrow gap semiconductor with a low effective mass and possesses the highest electron mobility among III-V compound semiconductors, which makes it very suitable for high-speed devices, magnetic sensors and so on $[2,3]$. InSbbased thin films are especially promising in the fabrication of long wavelength infrared detectors, and wavelengths greater than $12 \mu \mathrm{m}$ can be detected with these compounds based photodiodes at $77 \mathrm{~K}$ [5]. For the preparation of InSb thin layers on GaAs substrate, it is required to have special technological treatments to overcome the large lattice

\footnotetext{
* Corresponding author. Tel.: +8862 3366 3543; fax: +886 223677467.

E-mail address: zcfeng@cc.ee.ntu.edu.tw (Z. Chuan Feng).
}

mismatch of $\sim 14.6 \%$ between $\mathrm{InSb}$ and GaAs and to ensure their growth quality with specified electrical as well as optical properties of the layers $[3,4]$. There have been a number of studies on this material concerning the growth and optical characterization of InSb based compound semiconductors [6-8]. Efforts of the growth of these materials on GaAs substrate have been explored by various growth technique, such as molecular beam epitaxy (MBE) [3,4,912], liquid phase epitaxy (LPE) [13], magnetron sputter epitaxy [14], metalorganic vapor phase epitaxy (MOVPE) or metalorganic chemical deposition (MOCVD) [15-17]. But there lacks penetrating investigation on transport and lattice behaviors of these epitaxial materials.

Metalorganic vapor chemical deposition (MOCVD) technology has been shown a good technology to produce large size InSb thin film materials on GaAs substrate for industrial infrared and automobile applications [15-18]. 
In this paper, far-infrared (FIR) reflectance spectroscopy has been employed to study the lattice vibration behavior for a series of MOCVD-grown InSb films on GaAs substrates. From these MOCVD films, the effects of III$\mathrm{V}$ source ratios on the film crystalline quality have been investigated.

\section{Experimental}

The experimental MOCVD InSb epitaxial films were grown on commercial GaAs (100) substrates. Trimethyindium (TMIn) and Tris-dimethylamino-antimony (TDMASb) were used as In and $\mathrm{Sb}$ sources, respectively. The indium and antimony bubblers were operated at 429 and 323 Torr, respectively. Flows through the indium and antimony bubblers were varied from $130 \mathrm{sccm}$ to $270 \mathrm{sccm}$ and the carrier $\mathrm{H}_{2}$ gas flow were in the range of 320-775 sccm, respectively. Growth rates investigated were between 0.65 and $1.2 \mu \mathrm{m} / \mathrm{h}$ with an optimum growth rate of around $0.95 \mu \mathrm{m} / \mathrm{h}$. Growth temperature was monitored using a single wavelength low temperature pyrometer. Samples N01-08 were prepared at a III$\mathrm{V}$ ratio of between 4.2 and 4.5. Runs at these III-V values typically showed indium droplets on the surface of the film after growth. A second set of growth runs starting with run sample N09 was done using an III-V ratio of 6.2. These growths have resulted in surfaces with excellent morphology that were typically free of indium droplets. For all epitaxial InSb samples, a variety of characterization measurements have been performed, including surface morphology, Hall measurements, RF sheet resistivity, scanning electron microscopy (SEM), etc.

The far-infrared reflectance spectra were measured at near normal incident in far-infrared range, $60-500 \mathrm{~cm}^{-1}$, by a BRUKER IFS 120HR Fourier transform infrared (FTIR) spectrometer at different temperatures between 80 and $300 \mathrm{~K}$ with the spectral resolution better than 1 $\mathrm{cm}^{-1}$. Mercury-Arc lamp was used for infrared light source. A mirror-like gold plate was mounted next to the samples on the cold finger of cryogenic. The absolute reflectivity value of sample was determined by comparison with the gold mirror. An APD cryogenic system was employed to the temperature-dependent measurements. The temperature of finger tip inside the cryogenic

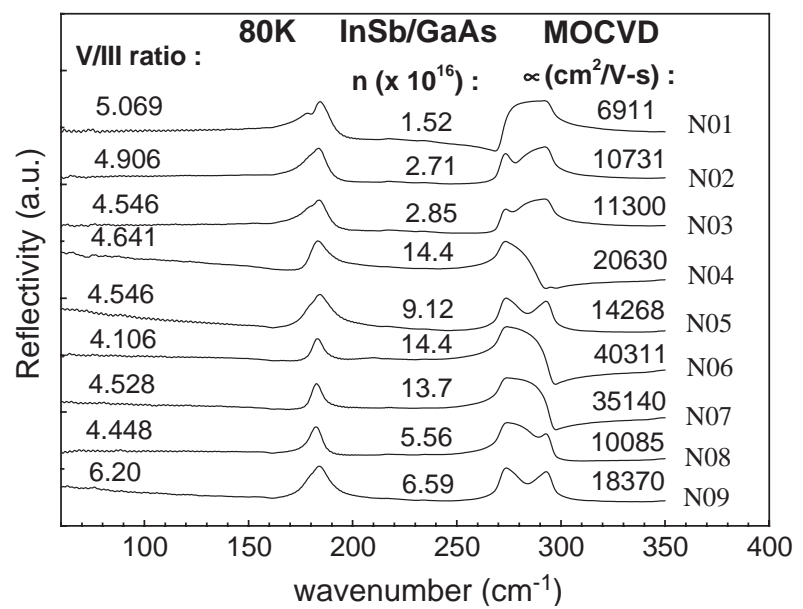

Fig. 1. FIR reflectance spectra of nine $\mathrm{InSb} / \mathrm{GaAs}$ samples, measured at $80 \mathrm{~K}$.

was controlled by a Lake-Shore 331 temperature controller with a temperature stability of $0.5 \mathrm{~K}$ or better.

\section{Results and discussion}

\subsection{Experimental far-infrared spectra of MOCVD films}

III-V source ratio has an important influence on the resulting epitaxial InSb films. Behet et al. [15] studied the dependence of InSb growth rate on III-V ratio in lowpressure $(20 \mathrm{~Pa})$ plasma MOVPE with triethylantimony (TESb) as Sb precursor. In this work, a series of InSb thin films were grown on GaAs under different III-V ratio conditions. The experimental values of III-V ratio and resulted sample surface Normaski microscopy and thickness uniformity are given in Table 1. Samples N01-N08 were grown with III-V ratio of 4.1-5.1. InSb films prepared at these III-V values typically showed indium droplets on the surface of the films after growth. Sample N09 was made using a III-V ratio of 6.2. This growth with a high III-V ratio had resulted in surfaces with excellent morphology that typically free of indium droplets. Hall measurements, sheet resistivity, SEM, and mid-infrared spectrum measurement for thickness showed a high thickness uniformity distribution, high mobility of the films which are very good for fast speed electron device applications.

Table 1

III - V ratio, surface morphology, thickness uniformity of samples

\begin{tabular}{|c|c|c|c|c|c|c|c|c|c|}
\hline Sample No & N01 & N02 & N03 & N04 & N05 & N06 & N07 & N08 & N09 \\
\hline III $-\mathrm{V}$ ratio & 5.069 & 4.906 & 4.546 & 4.641 & 4.546 & 4.106 & 4.528 & 4.448 & 6.20 \\
\hline Surface morphology & $\begin{array}{l}\text { In-rich/ } \\
\text { droplet }\end{array}$ & $\begin{array}{l}\text { Sb-rich/ } \\
\text { big lots }\end{array}$ & $\begin{array}{l}\text { In-rich/ } \\
\text { droplet }\end{array}$ & $\begin{array}{l}\text { Good/ } \\
\text { less drop }\end{array}$ & $\begin{array}{l}\text { Good/ } \\
\text { no drop }\end{array}$ & $\begin{array}{l}\text { In-rich/ } \\
\text { droplet }\end{array}$ & $\begin{array}{l}\text { Good/ } \\
\text { no-drop }\end{array}$ & $\begin{array}{l}\text { Sb-rich/ } \\
\text { less drop }\end{array}$ & $\begin{array}{l}\text { Good/ } \\
\text { no drop }\end{array}$ \\
\hline Thickness uniformity (\%) & 6.06 & 6.16 & 7.81 & 6 & 6.88 & 2.59 & 1.69 & 3.29 & 4.76 \\
\hline
\end{tabular}




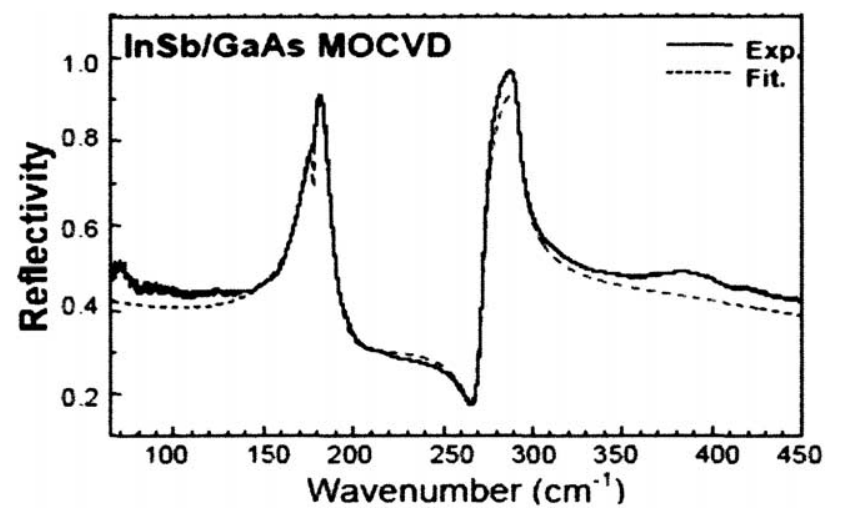

Fig. 2. Experimental (solid line) and theoretical (dash line) FIR reflectance spectra of InSb/GaAs, sample N01 at $300 \mathrm{~K}$.

A series of MOCVD-grown epitaxial InSb films on GaAs substrates were studied by FTIR method in the FIR region. The measured spectra of these samples $(80 \mathrm{~K})$ were shown in Fig. 1. The vibration modes of all these samples in these spectra are shown around $180 \mathrm{~cm}^{-1}$ and between 270 and $300 \mathrm{~cm}^{-1}$. Mode $183 \mathrm{~cm}^{-1}$ is assigned to the InSb TO phonon mode, and modes around $272-295 \mathrm{~cm}^{-1}$ are due to GaAs phonon modes from substrate.

\subsection{Theoretical simulation results for MOCVD InSb/GaAs}

The dielectric function of a semiconductor or layered structure, $\varepsilon(\omega)$, dependent on frequency $\omega$, can be described as $[19,20]$ :

$\varepsilon=\varepsilon_{\infty}+\sum_{j} \frac{S_{j} \omega_{\mathrm{TO} j}^{2}}{\omega_{\mathrm{TO} j}^{2}-\omega^{2}-i \gamma_{j} \omega}-\frac{\omega_{\mathrm{p}}^{2}}{\omega\left(\omega+i \gamma_{\mathrm{p}}\right)}$

where $\varepsilon_{\propto}$ is the dielectric function in high frequency limit, $\omega_{\mathrm{TO} j}$ and $\gamma_{\mathrm{TO} j}$ are the $j$ th transverse optical (TO) mode frequency and damping factor, $\omega_{\mathrm{p}}$ and $\gamma_{\mathrm{p}}$ are the free carrier plasma frequency and damping constant, respectively. The carrier concentration, $n$, and mobility, $\mu$, can be obtained from:

$\omega_{\mathrm{p}}^{2}=\frac{n e^{2}}{m^{*} \varepsilon_{\infty} \varepsilon_{0}}$ and $\gamma_{\mathrm{p}}=\frac{e}{m^{*} \mu}$,

where $e$ is the electron charge, $m^{*}$ is the electron effective mass and $\varepsilon_{0}$ is the vacuum dielectric constant. Based upon these theoretical equations, simulation can be performed to fit to all experimental FIR spectra in the long wavelength limit. A multi-layers ( $\mathrm{InSb}+\mathrm{GaAs}$ two layers) fitting technique was applied in theses theoretical calculation. Contributions of inner shell dielectric value and a set of Lorentzian vibrators as well as carrier contribution and also the contribution from GaAs substrate are included. A typical fitted datum is shown in Fig. 2. The mode of around $180 \mathrm{~cm}^{-1}$ by least square fit is assigned as InSb TO mode and the mode around $270-300 \mathrm{~cm}^{-1}$ was fitted with different InSb film thickness and assigned as GaAs TO mode in $270 \mathrm{~cm}^{-1}$ at $300 \mathrm{~K}$. All of the phonon modes assignments of these samples were calculated and fitted by dielectric response model. The fitted optical parameters of all these $\mathrm{InSb}$ films at $300 \mathrm{~K}$ were given in Table 2.

\subsection{The temperature dependence of FIR spectra on InSb/ GaAs}

The variation of temperature can cause the above modes blue-shifted. The temperature-dependent FIR spectra of all these samples have been measured and well fitted (not shown here). The results showed that the InSb TO mode has about $3 \mathrm{~cm}^{-1}$ blue shift from temperature at $300 \mathrm{~K}$ down to $80 \mathrm{~K}$, and that the GaAs TO mode from the substrate has about $1-2 \mathrm{~cm}^{-1}$ blue shift mostly. The wavenumber values of $\mathrm{TO}$ modes of $\mathrm{InSb}$ and $\mathrm{GaAs}$ of all these samples are corresponding to those calculated according to the Kramers-Kroning $(\mathrm{K}-\mathrm{K})$ relation of reflection spectrum.

\subsection{Carrier concentration and mobility}

Carrier concentration and sheet resistance are critical for device applications. The values of carrier concentration and mobility are dependent on sample growing conditions. The fitted values of carrier concentration in the present $\mathrm{InSb} /$ GaAs samples are varied between $10^{16}$ and $10^{17} \mathrm{~cm}^{-3}$ at $300 \mathrm{~K}$ as shown in Table 2. These values are about the same order as that from Hall measurement with a very slight difference. The sample with higher carrier concentration has higher absolute reflectance intensity in the long wavelength range as shown in Fig. 1. The higher reflection intensity in the long wavelength range is due to the plasma effect and also due to that InSb has a very narrow band gap which

Table 2

Samples optical parameters were fitted by dielectric response model in the long wavelength limit

\begin{tabular}{lllllllcrc}
\hline Sample & $\begin{array}{l}\text { III-V } \\
\text { ratio }\end{array}$ & $\varepsilon_{8}$ & $\begin{array}{l}\omega_{\text {TO }} \\
\left(\mathrm{cm}^{-1}\right)\end{array}$ & $\begin{array}{l}\text { Strength } \\
\mathrm{S}\end{array}$ & $\begin{array}{l}\text { Damping } \\
\Gamma\left(\mathrm{cm}^{-1}\right)\end{array}$ & $\begin{array}{l}\text { Carrier concentration } \\
\left(\times 10^{16} \mathrm{~cm}^{-3}\right)\end{array}$ & $\begin{array}{l}\text { Mobility } \\
\left(\mathrm{cm}^{2} / \mathrm{V} \mathrm{s}\right)\end{array}$ & $\begin{array}{l}\text { Effective } \\
\text { mass }\end{array}$ & $\begin{array}{l}\text { Thickness } \\
(\mu \mathrm{m})\end{array}$ \\
\hline N01 & 5.069 & 17.88 & 179.3 & 2.5 & 1.3 & 1.52 & 6911 & 0.0129 \\
N02 & 4.906 & 17.88 & 178.7 & 2.4 & 3.3 & 2.71 & 10730 & 0.0170 \\
N03 & 4.546 & 17.88 & 178.4 & 2.5 & 1.6 & 2.85 & 11300 & 0.0173 \\
N04 & 4.641 & 17.88 & 179.5 & 3.2 & 4.7 & 1.44 & 20630 & 0.0152 \\
N05 & 4.546 & 17.88 & 180.5 & 2.4 & 1.5 & 9.12 & 14268 & 0.0186 \\
N06 & 4.106 & 17.88 & 180.1 & 1.9 & 4.0 & 1.44 & 40311 & 0.0176 \\
N07 & 4.528 & 17.88 & 178.8 & 2.0 & 3.1 & 1.37 & 35140 & 0.0177 \\
N08 & 4.448 & 17.88 & 178.3 & 2.0 & 2.5 & 5.56 & 1.57 & 0.66 \\
N09 & 6.20 & 17.88 & 180.5 & 2.5 & 4.2 & 6.59 & 18370 & 0.0135 \\
\hline
\end{tabular}


enhances the plasma effect as long as the carrier concentration become higher.

\subsection{Extra modes due to interface reaction}

The large lattice mismatch $(\sim 14.6 \%)$ between the InSb Film and GaAs substrate is accompanied by a high density of dislocations and defects between the interface of the film that can deteriorate the quality of thin film [21]. Because of the longer penetration depth in the long wavelength range of FIR spectra, in Fig. 3 and insert, we can clearly see the third and fourth small vibration temperature-dependent modes appear in around $218 \mathrm{~cm}^{-1}$ and $228 \mathrm{~cm}^{-1}$. These modes were due to the interface reaction between film and substrate and observed obviously in the lower temperature of $80-150$ $\mathrm{K}$ as indicated in the three upper curves in Fig. 3. These vibration modes were assigned as InAs and $\mathrm{GaSb}$ TO modes [22]. Also we see these modes having blue shift of measured spectra as temperature decreases.

\subsection{InSb film thickness}

The determination of InSb epitaxial film thickness and distribution over the entire layer is critical in the epilayer growth, especially in the large wafer diameter production. A scanning electron microscope (SEM) was usually employed to measure the InSb film thickness $[16,18]$. But, it is destructive and not convenient for industrial production. The reflectance fringes do not appear in the ultraviolet and visible range from the $\mathrm{InSb} / \mathrm{GaAs}$ heterostructure. However, the reflectance fringes from $\mathrm{InSb} / \mathrm{GaAs}$ can be observed in the mid-near infrared range [17]. We have found that it is

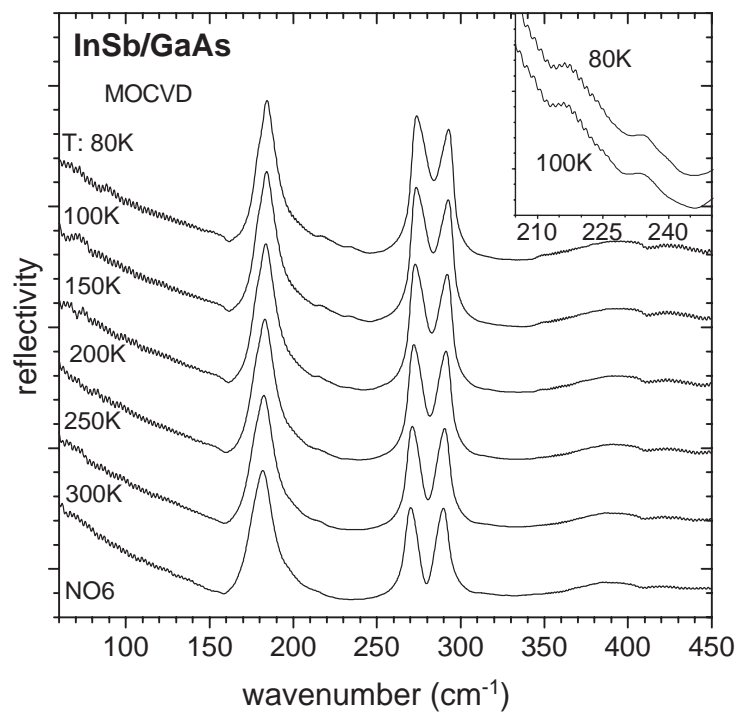

Fig. 3. FIR reflectance spectra of InSb/GaAs sample N06 at 80-300 K with the spectral line shifted up as the temperature increases. The InSb TO mode has about $3 \mathrm{~cm}^{-1}$ blue shift from temperature at $300 \mathrm{~K}$ down to $80 \mathrm{~K}$. The GaAs mode from the substrate has about $1-2 \mathrm{~cm}^{-1}$ blue shift from temperature at $300 \mathrm{~K}$ down to $80 \mathrm{~K}$. The insert shows two weak modes appeared at low temperature of $80-100 \mathrm{~K}$.

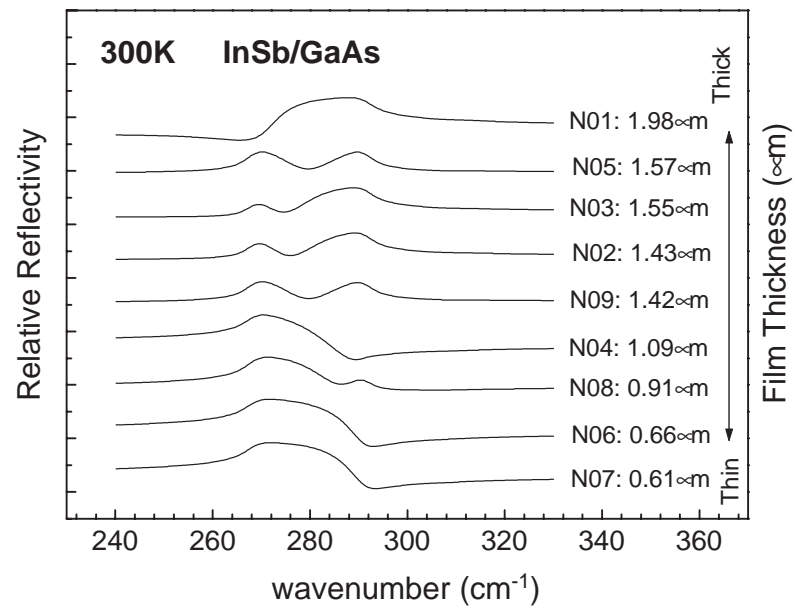

Fig. 4. FIR reflectance spectra, between 240 and $330 \mathrm{~cm}^{-1}$, of nine $\mathrm{InSb} /$ GaAs samples, listed in the order of the film thickness. The TO mode in around $270 \mathrm{~cm}^{-1}$ is well known as the GaAs mode which is contributed from the film substrate. The film thickness by fitted values is shown in the figure. We use the GaAs TO modes $\left(\sim 270 \mathrm{~cm}^{-1}\right.$ at $\left.300 \mathrm{~K}\right)$ instead of two modes to well fit the splitting of this GaAs mode.

easy to observe the interference fringes in far-infrared range of $60-500 \mathrm{~cm}^{-1}$ or $20-160 \mu \mathrm{m}$. In this study, we have obtained the InSb film thickness by experimental datum fitting. The contribution of InSb film absorption to its dielectric value in the long wavelength range limit was calculated with respect to different film thicknesses, and the obtained values were used to fit the experimental datum. The results of fitted film thickness were shown in Table 2.

\subsection{The splitting of GaAs TO mode}

From Figs. 1, 3 and 4), an interesting phenomenon can be found, i.e., the splitting of the GaAs TO mode in the FIR

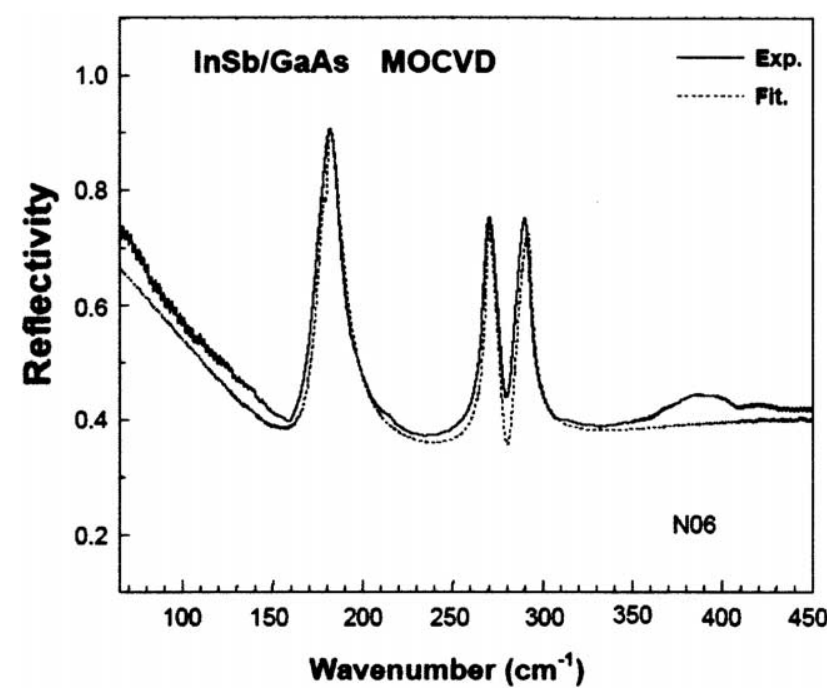

Fig. 5. FIR spectrum and fits of MOCVD InSb/GaAs, N06, at $300 \mathrm{~K}$. The mode at $180 \mathrm{~cm}^{-1}$ is assigned for the InSb TO phonon mode, and the mode around $270-290 \mathrm{~cm}^{-1}$ is assigned for the GaAs phonon mode from substrate. The split of this GaAs mode is assuming due to the contribution of absorption of different InSb film thickness. 
spectra from some InSb/GaAs samples but not in some other samples. In Fig. 3, the TO mode in around $270 \mathrm{~cm}^{-1}$ is well known as GaAs mode which is contributed from the film substrate. The splitting of this GaAs mode could clearly be seen in the FIR spectrum and can be explained due to the variation of the InSb film thickness. We use the GaAs TO mode $\left(\sim 270 \mathrm{~cm}^{-1}, 300 \mathrm{~K}\right)$ instead of two modes with varying the thickness of InSb film to fit all these FIR spectra in this range. Fig. 5 shows such an example with the experimental spectrum and its fitting. The variation of $\mathrm{InSb}$ film thickness from our dielectric response fitting is found to be exactly the factor contributed to this mode splitting as observed in Figs. 4 and 5.

\section{Conclusion}

We had performed the optical characterization on a series of InSb thin films grown on GaAs by MOCVD under different growth conditions by way of far-infrared spectroscopy and theoretical simulation. Various mode parameters, e.g., the TO mode frequency, carrier concentration, mobility, etc., were obtained from these $\mathrm{InSb} / \mathrm{GaAs}$ materials. All the specimens were analysed by a dielectric response model. It was found that the extra modes appear in FIR spectra due to the interface reaction between the film and substrate. Interesting splitting of the GaAs transverse optical phonon mode was observed and explained using the dielectric response model to fit the FIR reflectance spectrum by varying the InSb film thickness. Effects of III-V source ratios were successfully studied by FIR spectroscopy and the optimized growth parameters have been obtained. The InSb film grown with an III-V ratio of 4.1 possesses the highest mobility and nearly lowest carrier concentration. The results of this study show that MOCVD technology is capable to produce high quality far-infrared InSb epitaxial materials, and the FTIR method is a very useful tool for non-destructive characterization of large size wafers in industrial mass production.

\section{Acknowledgement}

We acknowledge the support, materials preparation and help in this work from Drs. N. Schumaker, R. Stall, I.
Ferguson, and C. Beckham. The work at National Taiwan University was supported by funds from National Science Council of Republic of China, NSC 93-2218-E-002-011 and 93-2215-E-002-035.

\section{References}

[1] V.K. Dixit, B.V. Rodrigues, H.L. Bhat, R. Kumar, R. Venkataraghavan, K.S. Chandrasekaran, B.M. Arora, J. Appl. Phys. 90 (2001) 1750.

[2] V.K. Dixit, B.V. Rodrigues, H.L. Bhat, R. Venkataraghavan, K.S. Chandrasekaran, B.M. Arora, J. Cryst. Growth 235 (2002) 154.

[3] T. Zhang, S.K. Clowers, M. Debnath, A. Bennett, C. Roberts, J.J. Harris, R.A. Stradling, L.F. Cohen, Appl. Phys. Lett. 84 (2004) 4463.

[4] T.D. Mishima, M.B. Santos, J. Vac. Sci. Technol., B 22 (2004) 1472.

[5] A. Cappy, B. Carnez, R. Fauguemergues, G. Salmer, E. Constant, IEEE Trans. Electron Devices ED-27 (1980) 2158.

[6] J.R. Sendra, G. Armelles, T. Utzmeier, J. Anguita, F. Briones, J. Appl. Phys. 79 (1996) 8853.

[7] J. Wagner, J. Schmitz, Philos. Mag., B 70 (1994) 467.

[8] R. Addinall, R. Murry, R.C. Newman, J. Wagner, S.D. Parker, R.L. Williams, R. Droopad, A.G. DeOliveira, I. Ferguson, R.A. Stradling, Semicond. Sci. Technol. 6 (1991) 147.

[9] E. Michel, G. Singh, S. Slivken, C. Besikci, P. Bove, et al., Appl. Phys. Lett. 65 (1994) 3338.

[10] P.K. Chiang, S.M. Bedair, J. Electrochem. Appl. Phys. Lett. 46 (1995) 383.

[11] R.M. Biefeld, G.A. Hebner, Appl. Phys. Lett. 57 (1990) 1563.

[12] K. Kanisawa, H. Yamaguchi, Y. Hirayama, Appl. Phys. Lett. 76 (2000) 589.

[13] D.E. Holmes, G.S. Kamath, J. Electron. Mater. 9 (1980) 95.

[14] J. Webb, R. Rousina, in: Z.C. Feng (Ed.), Semiconductor Interfaces and Microstructures, World Scientific, Singapore, 1992, p. 199.

[15] M. Behet, B. Stoll, W. Brysch, K. Heime, J. Cryst. Growth 124 (1992) 377.

[16] B.-S. Yoo, M.A. McKee, S.-G. Kim, E.-H. Lee, Solid State Commun. 88 (1993) 447.

[17] Z.C. Feng, C. Beckham, P. Schumaker, I. Ferguson, R.A. Stall, N. Schumaker, M. Povloski, A. Whitley, in: F. Julien, T. Myers, M. Manasreh (Eds.), Infrared Applications of Semiconductors-Materials, Processing, and Devices, Mater. Res. Soc. Symp. Proc., vol. 450, MRS, Pittsburgh, 1997, pp. 61-66.

[18] M.A. Mckee, B.-S. Yoo, R.A. Stall, J. Cryst. Growth 124 (1992) 286.

[19] S. Perkowitz, Optical Characterization of Semiconductors, Academic Press, San Diego, 1993, p. 39.

[20] T.R. Yang, C.C. Lu, W.C. Chou, Z.C. Feng, S.J. Chua, Phys. Rev., B 60 (1999) 16058.

[21] Z.C. Feng, S. Perkowitz, T.S. Rao, J.B. Webb, J. Appl. Phys. 68 (1990) 5363.

[22] C. Kittel, Introduction to Solid State Physics, 7th edition, John Wiley \& Son, Inc., New York, 1996, p. 292. 